Journal of Systems Science and Information

Feb., 2015, Vol. 3, No. 1, pp. 25-36

DOI: $10.1515 /$ jssi-2015-0025

\title{
A Dynamic Clustering Method to Large-Scale Distribution Problems
}

\author{
Zhizhong TANG \\ College of Management and Economics, Tianjin University, Tianjin 300072, China \\ E-mail: tangzhizhong@tfl-tj.com \\ Bo LI \\ College of Management and Economics, Tianjin University, Tianjin 300072, China \\ E-mail: libo0410@tju.edu.cn \\ Hongyan QIU \\ College of Management and Economics, Tianjin University, Tianjin 300072, China \\ E-mail: qhy137@126.com
}

\begin{abstract}
This paper presents the dynamic fuzzy clustering method to solve the multi-producers to multi-customers large-scale distribution problem. The proposed method includes three phases: Static clustering, order processing, and dynamic clustering. Based on the distances among customers, $k$ means method is used to generate the static clusters. The service priorities of each producer serving the static customer groups are ranked according to the distance performance. In the case of fluctuant customer orders, order processing can divide customer orders into several consecutive periods. After the above two phases, the fuzzy clustering technique is applied to further conduct dynamic clustering based on the customer order attributes. Similarly, the service priorities of generated dynamic customer groups will be ranked according to the time attributes of orders. Finally, by the real case, the authors obtain the conclusion that using the proposed method, the total cost of the producer is reduced by about $35 \%$, and the vehicle loading rates are almost above $95 \%$.
\end{abstract}

Keywords large-scale distribution scheduling; fluctuant orders; fuzzy clustering; dynamic groups

\section{Introduction}

In recent years, more and more companies organize their production lines in the areas which are rich in resources or customer centralized, while the distribution scope of customers is gradually expanding. Then there appears a large-scale distribution network between multiproducers and multi-customers. At the same time, the customer demands are increasingly diverse and the delivery deadlines required by customers are more stringent. Thus, how to respond to fluctuant customer orders quickly, integrate the logistics resources, and improve the service level are major challenges to most companies. Our study is motivated by the case of one company, a large-scale milk producer in China, but our contribution is of general applicability.

The literature on studying a large-scale distribution problem is fruitful. A typical way to solve a large-scale distribution problem is to partition the customers into groups first and then

Received January 24, 2014, accepted June 19, 2014

Supported by the Specialized Research Fund for Doctoral Program of Higher Education, China (20100032110034) 
construct optimal routes for each group or groups. Conventionally, customers are grouped just according to the geographical distance, while the customer demand attributes are ignored. For example, Li and Jiang ${ }^{[1]}$ took the distribution period and distribution distance respectively as the criterion to classify customers. Zhu and $\mathrm{Li}^{[2]}$ developed a new static clustering method based on Artificial Immune System considering the geographical distances among customers. Yang et al. ${ }^{[3]}$ extended a new variant of capacitated clustering problem and proposed a Lagrangian relaxation approach (LR) with two phases of dual optimization. Nananuku[ ${ }^{[4]}$ studied a reactive Tabu search for solving the customers' clustering problem by analyzing the key factors, including demand pattern, holding costs, etc.

Additionally, there are also some references that apply the fuzzy clustering method to solve the distribution problem. Lu et al. ${ }^{[5]}$ used fuzzy clustering analysis to classify demand points on the basis of physical distribution. $\mathrm{Hu}$ and Sheu ${ }^{[6]}$ stressed that customer classification was important for the development of advanced logistical distribution and used the fuzzy clustering method to cluster customers before executing fleet routing. Sheu ${ }^{[7]}$ proposed a dynamic customer group-based logistics resource allocation methodology for the city logistics distribution operations which mainly used the fuzzy clustering method. A new fuzzy clustering method was explored by Kuo et al. ${ }^{[8]}$, which included three steps: Binary transformation, generation of a fuzzy correlation matrix, and customer clustering. Sheu ${ }^{[9]}$ presented an integrated fuzzyoptimization customer grouping method for responding to a variety of customer demands. The fuzzy clustering technique was also provided for solving large scale stochastic vehicle routing problems $^{[10]}$. Qi et al. ${ }^{[11]}$ proposed a new measurement method for customers partitioning which was based on both spatial location and service time window. A genetic algorithm based on clustering technique was applied to solve the multi-depot vehicle routing problem ${ }^{[12]}$. Wang et al. ${ }^{[13]}$ developed a fuzzy-based customer clustering algorithm with a hierarchical analysis structure to address customer clustering problem. However, the backgrounds of the above references mostly focus on one producer (distribution center) to multi-customers. Yin et al. ${ }^{[14]}$ organized an approach to cluster the supply chain network and fuzzy $c$-means was proposed to solve the problem. Most of them just consider the static situation and lack the consideration about the fluctuant orders processing, which can not be well applied in real-world scenarios ${ }^{[15,16]}$.

This paper proposes a dynamic fuzzy clustering method to solve the multi-producers to multi-customers in the large-scale distribution scheduling problem faced by the large companies. The method takes delivery distance and the customer order attributes into consideration simultaneously. The remainder of the paper is organized as follows. Section 2 illustrates the overall methodology framework. The detailed solution methodology is described in Section 3, including static clustering, order processing, and dynamic clustering. Section 4 mainly discusses the routes construction and comparison between the proposed method and the direct distribution, followed by conclusion in Section 5 .

\section{Model Descriptions}

Assuming that $G=(V, A)$ is a graph, where $V=P \cup C$ is a set of points, and $P, C$ are the sets of producers and customers respectively. $A=\{(i, j): i, j \in V, i \neq j\}$ is the set of routes between two points. During the same scheduling period, the quantities and the kinds of 
the products are known and we suppose that the outbound logistics is JIT in the distribution center of every producer, so the holding cost is not considered here.

To simplify the computation, during the scheduling period $t \in T$, a fleet of vehicles with the same capacity $Q$ is assumed and a request or multi-requests from customers are satisfied by one vehicle or multi-vehicles dispatched from the distribution centers, $T$ is the total scheduling period. Because the requests from customers may concern with many kinds of products, and the total amount of all demands from a customer may be less than a truck load, so it leads to a high cost if the requests from customers are not integrated and loaded into the trucks. Therefore, the goal of this paper is to optimize a distribution problem which includes multi-producers, multi-kinds of products, multi-customers in a large scale logistics system.

The notations and parameters are defined as follows: $P$ is a set of $p$ producers. $C$ is a set of $n$ customers. $I$ is a set of $i$ kinds of products. $K$ is a set of $k$ vehicles. $Q_{i p c t}$ represents the quantities from the producer $p$ to satisfy the requests of a customer $c$ for the product $i$ during the scheduling period $t . d_{p c}$ denotes the distance from the producer $p$ to the customer $c . F_{p c}$ is the shipping cost per unit distance of products from the producer $p$ to the customer $c$. During the scheduling period $t, T Q_{i p t}$ represents the total production capacity of the producer $p$ on the product $i$. $S_{i p t}$ denotes the quantities of the product $i$ satisfied by the producer $p$. $Q$ is the capacity of every vehicle. $M$ denotes a very big constant. $X_{i p c k t}$ is defined as a decision variable, if the requests on the product $i$ from the producer $p$ to the customer $c$ is satisfied by the vehicle $k$, then $X_{i p c k t}=1$, otherwise $X_{i p c k t}=0 . Z_{p c t}$ is the other decision variable and if the producer $p$ is asked to satisfy the customer $c$, then $Z_{p c t}=1$, otherwise $Z_{p c t}=0$.

Thus, the model is given as follows:

$$
\begin{aligned}
\min & \sum_{t \in T}\left\{\sum_{p \in P} \sum_{c \in C}\left(\sum_{k \in K} \sum_{i \in I} Q_{i p c t} \cdot X_{i p c k t}\right) \cdot d_{p c} \cdot F_{p c}+M \cdot\left(\sum_{p \in P} \sum_{c \in C} Z_{p c t}\right)\right\} \\
\text { s.t. } & \sum_{c \in C} Q_{i p c t} Z_{p c t} \leq T Q_{i p t}, \quad \forall i \in I, \forall p \in P, \forall t \in T \\
& \sum_{k \in K} \sum_{p \in P} Q_{i p c t} \cdot X_{i p c k t} \leq \sum_{i \in I} T Q_{i p t} Z_{p c t}, \quad \forall p \in P, \forall c \in C, \forall t \in T \\
& \sum_{i \in I} Q_{i p c t} \cdot X_{i p c k t} \leq Q, \quad \forall p \in P, \forall c \in C, \forall k \in K, \forall t \in T \\
& Q_{i p c t} X_{i p c k t} \leq S_{p i} Z_{p c t}, \quad \forall p \in P, \forall c \in C, \forall k \in K, \forall i \in I, \forall t \in T \\
& X_{i p c k t} \in\{0,1\}, \quad Z_{p c t} \in\{0,1\}
\end{aligned}
$$

This is a multi-objective problem. The objective in formula (1) means that during the delivery from the producer $p$ to the customer $c$ in the scheduling period $t$, the first term is the shipping cost of all vehicles $k$, the second term minimizes the number of the producers which can provide every product $i$ to a customer $c$. The constraint (2) represents that the total weight of the product $i$ satisfied by every producer equals to or less than its supply capacity. The constraint (3) means that the weight of the product requested by a customer $c$ equals to or less than the supply amount of all producers which have the ability to produce the product $i$. The constraint (4) ensures that the delivery weight cannot exceed the capacity of every vehicle. The constraint (5) ensures that if a vehicle $k$ is dispatched to visit a customer $c$ with 
the product $i$, then there exist the producers to product this product. The constraints (6) are the variable constraints valued by 0,1 .

\section{A Three-Phase Hybrid Solving Method}

In this section we provide the detailed description of the proposed solution methodology. The solution methodology includes order processing and clustering. The order processing is to determine which orders should be served in the current service period, and this phase is primarily taking the lead time, the time of order entry, the delivery deadline and the service period length as input variables. The objective of clustering is to divide a complex problem into several easily solved sub-problems. Firstly, we give the proposed methodology framework.

\subsection{The Framework}

To solve the above model, we develop a three-phase solution methodology framework capable of quickly generating practical solutions to this very complicated problem faced by the company, as shown in Figure 1. In the first phase, we use $k$-means clustering method to form static customer clusters which are based on the distance between each pair of customers, and then the service priorities from each producer to the static customer groups are ranked according to the distance performance. The second phase is order processing that aims to determine the target customer orders which are processed and served in a given service period. The resulting outputs of the above two phases are inputted to the final phase in which the fuzzy clustering technique is used to further form dynamic clusters based on the customer order attributes. Similarly, the generated dynamic customer groups will be ranked according to the time attributes of orders. Finally, optimal routes are constructed in every dynamic group by minimizing the total travel distance cost. The last two phases are carried out each time when there are new arrival orders, whereas those static customer clusters remain unchanged in the long run because that company always has the regular customers in a relatively stable period.

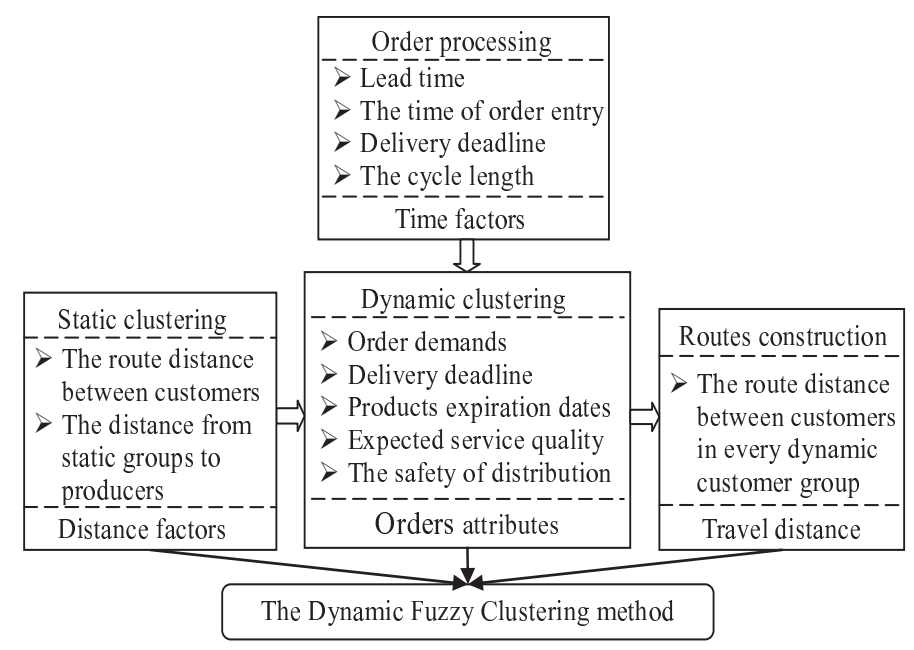

Figure 1 The proposed methodology framework 
In static clustering stage, a large set of customers are clustered into several static groups by employing the $k$-means cluster method based on the real delivery distances among customers. At the same time, every generated static group is assigned to each producer depending on the distance among them, which means that the complicated multi-to-multi problem can be divided into many simple one-to-multi sub-problems. It is of great importance to measure the distance between each pair of customers. After generating the static groups, the company should reserve at least $m$ vehicles for each static group depending on the total customer demands of the static group. $m$ is calculated from Equation (7), where $T D$ denotes the total customer demands of the static group, and $Q$ is the vehicle capacity.

$$
m=\left\lfloor\frac{T D}{Q}\right\rfloor+1
$$

\subsection{Order Processing}

To facilitate model formulation, it is assumed that the period of customer order processing is fixed, and it is equal to the scheduling period $t$. In addition, we conduct vehicle routing dispatching once in each service period $t$. Note that the length of $t$ may depend on the operational conditions of companies. Supposing that: The order entry time of customer $i$ is $t$, and the delivery deadline of customer $i$ is $\bar{t}_{i}, \underline{t}$ and $\bar{t}$ respectively represent the start and end of the service period $t, \underline{L}$ and $\bar{L}$ are defined as the allowable minimum and maximum lead times that the company commits to customers. Those time variables should satisfy the following constraints:

$$
\begin{aligned}
& \underline{L} \leq \underline{t}-\overline{t_{i}} \leq \bar{L} \\
& \bar{L} \leq \overline{t_{i}}
\end{aligned}
$$

Equation (8) denotes the upper bound of the lead time associated with a given customer $i$, and Equation (9) ensures that the corresponding delivery deadline is not violated. Arrival orders should be processed firstly at the beginning of one processing period. Meanwhile, if one order doesn't meet the above conditions, it will be processed in the next service period. As the processing period is continuous, the customer orders can be processed real-timely. So, the company can respond to customer demands more quickly, and thus the customer satisfaction will be greatly enhanced.

\subsection{Dynamic Clustering}

The purpose of this phase is using the fuzzy clustering technique to generate dynamic distribution groups. We should define order attributes firstly, and then transform them into triangular fuzzy numbers for calculating the similarity between them, which is based on the proposed five linguistic terms in this paper. Finally, in each static group, customers with higher similarity are further clustered into the same dynamic group. Considering the orders' time attributes, it is necessary to further calculate the service priority of dynamic groups, which is combined with the producer service priority mentioned in static clustering to determine whether the producer serve the dynamic group. If the producer with the first top service priority cannot meet the demands of one dynamic group, then the producer with the second priority is selected, and the rest is decided by the same analogy. 
1. Order attributes definition

The defined orders attributes are the dynamic clustering decision variables, including order demands, the delivery deadline, products expiration dates, expected service quality, and the safety of distribution. The first three are quantitative variables, while the latter two are qualitative variables. In addition, delivery deadline and products' expiration dates are the time attributes of orders, which determine the respective service priority of every dynamic group. The specific descriptions of those attributes are analyzed as follows:

1) $y_{i}^{1}$ represents the order demands of the customer $i$. As mentioned in Section 1 , the company has many small customers, which always bring more LTL transportation requirements and lower vehicle loading rates. So we should integrate the customer order demands by clustering, and yield the dynamic groups.

2) $y_{i}^{2}$ is the delivery deadline variable of the customer $i$. In real-world operations, the delivery deadline must not be violated, and it is necessary to deliver products to those customers with close distribution deadlines by the same fleet. And thus, these customers can be clustered into one dynamic group.

3) $y_{i}^{3}$ denotes the expiration dates of products that the customer $i$ orders. In fact, customers often pay too much attention to the products' market value or quality. Therefore, those products with the similar expiration dates tend to be delivered together in the distribution operations.

4) $y_{i}^{4}$ shows the service quality expected by the customer $i$. It is generally agreed that the service quality depends on the response time to customer demands as well as the delivery service attitude. The service quality determines the customer satisfaction to the company. From a practical point of view, the company can cluster those customers into the same dynamic group who demand similar service quality for providing different delivery services.

5) $y_{i}^{5}$ is defined as the security requirements of distribution process. In fact, customers always concern about the security of distributed products. To a great extent, this reflects a customer's personal demands in terms of condition of the product. In order to satisfy customers' specific needs for delivery security, the company should group customers with similar security requirements together to delivery.

2. Triangular fuzzy number transformation

After defining the above five variables, each customer order can be expressed as a multiattribute set. We use five linguistic terms to measure the decision variables, which are "very high", "high", "medium", "low", and "very low", and every linguistic term corresponds to a triangular fuzzy number, as shown in Table 1 . Such as: "( $0,0.75,1)$ " represents the linguistic item "very high". Therefore, in static group $g$, the given orders attribute $k$ of the customer $i$ can be transformed into a triangular fuzzy number with three items $\left(\sigma_{i \theta}^{k}\right)$, which can be expressed as follows:

$$
\tilde{y}_{i \theta}^{k}=\left(\sigma_{i \theta, 1}^{k}, \sigma_{i \theta, 2}^{k}, \sigma_{i \theta, 3}^{k}\right)
$$

Generally, in the company, the delivery deadlines comprise 1day, 2days, 3days, 4days, and 5days. There are five kinds of products expiration dates, including 30days, 45days, 60days, 90days, and 180days. We can transform them into triangular fuzzy numbers sequentially, for example, one customer order attributes are (3.1t, 2days, 60days, high, very high), and the corresponding triangular fuzzy numbers are $[3.1 \mathrm{t} ;(0,0.25,0.5) ;(0.25,0.5,0.75) ;(0.5,0.75,1)$; 
$(0.75,1,1)]$. Additionally, the order demand variable combined with the vehicle capability is the key factor to determine whether to stop the dynamic clustering, that is to say, it does not need similarity evaluation.

Table 1 The triangular fuzzy number of five linguistic terms

\begin{tabular}{llll}
\hline & \multicolumn{3}{c}{ triangular fuzzy number } \\
\cline { 2 - 4 } Linguistic terms & $\sigma_{i \theta, 1}^{k}$ & $\sigma_{i \theta, 2}^{k}$ & $\sigma_{i \theta, 3}^{k}$ \\
\hline Very high & 0.75 & 1 & 1 \\
High & 0.5 & 0.75 & 1 \\
Medium & 0.25 & 0.5 & 0.75 \\
Low & 0 & 0.25 & 0.5 \\
Very low & 0 & 0 & 0.25 \\
\hline
\end{tabular}

\subsection{Similarity Measuring and Clustering}

At this stage, for each static group $g$, a $n^{g} \times n^{g}$ fuzzy similarity matrix $Z^{g}$ is constructed in which each element $z_{i^{g} j^{g}}$ denotes the similarity between a given pair of customers $i^{g}$ and $j^{g}$. The fuzzy similarity matrix $Z_{g}$ is shown as follows:

$$
Z^{g}=\left[\begin{array}{cccc}
z_{11} & z_{12} & \cdots & z_{1 n^{g}} \\
z_{21} & z_{22} & \cdots & z_{2 n^{g}} \\
\vdots & \vdots & \ddots & \vdots \\
z_{n^{g}} & z_{n^{g} 2} & \cdots & z_{n^{g} n^{g}}
\end{array}\right]
$$

The element $z_{i^{g} j^{g}}$ is calculated by using Equations (12) (14), where $S\left(\tilde{y}_{i^{g}}^{k}, \tilde{y}_{j^{g}}^{k}\right)$ is the similarity of customers $i^{g}$ and $j^{g}$ on the attribute $k$, and obtained from Equation $(7)^{[10,11]} \cdot w_{k}$ denotes the weight coefficient of attribute $k$. In our study, parameters $\left(w_{2}, w_{3}, w_{4}, w_{5}\right)$ are set as $(0.3$, $0.2,0.3,0.2)$. Parameters' setting depends on the survey data that the company collects from its customers.

$$
\begin{aligned}
& z_{i^{g} j^{g}}=\sum_{k=2}^{5} \omega_{k} S\left(\tilde{y}_{i^{g}}^{k}, \tilde{y}_{j^{g}}^{k}\right) \\
& \sum_{k=2}^{5} \omega_{k}=1, \quad \omega_{k} \geq 0, \quad k=2,3,4,5 \\
& S\left(\tilde{y}_{i^{g}}^{k}, \tilde{y}_{j^{g}}^{k}\right)=1-\left(\sum_{t=1}^{3}\left|\sigma_{i^{g}, t}^{k}-\sigma_{j^{g}, t}^{k}\right|\right) / 3
\end{aligned}
$$

Based on the generated fuzzy similarity matrix, customers with higher similarity are assigned to the same dynamic group. The steps of dynamic clustering are described as follows:

Step 1 In the matrix $Z^{g}$, given a target customer $j^{g}$, remove the row $Z_{j^{g}}^{\mathrm{T}}$ associated with customer $j^{g}$. 
Step 2 Find the maximum element in the column $Z_{j^{g}}$, denoted by $z_{i^{g} j^{g}}$, and then conduct the following procedures:

1) If the condition $z_{i^{g} j^{g}}>\lambda$ holds, and the total order demands of this dynamic group do not exceed the $m$ times of the vehicle capacity after adding the customer $i^{g}$, then assign customer $i^{g}$ to the same group with customer $j^{g}$. Remove the row associated with customer $i^{g}$.

2) Go back to Step 2; continue to check the other elements of column $Z_{j^{g}}$ until no element satisfies the condition.

3) Remove the column $Z_{j^{g}}$.

Step 3 If there are any customers unassigned at this stage, then go back to Step 1 until all customers are processed.

Here, $\lambda$ is the criterion that identifies the relative similarity among customers. $\lambda$ determines the number of iteration steps and the number of clustering groups, which is generally greater than 0.5. In this study, $\lambda$ is set to be 0.75 .

According to the time attributes: Delivery deadline and products expiration dates, this step is to determine the service priority $l_{g_{i j}}$ of dynamic group $g_{i j}$ using Equation (15).

$$
\begin{aligned}
& l_{g_{i j}}=\omega_{2}^{\prime} \frac{\sum_{i=1}^{n} y_{i^{g}}^{2}}{n}+\omega_{3}^{\prime} \frac{\sum_{i=1}^{n} y_{i^{g}}^{3}}{n} \\
& \omega_{2}^{\prime}+\omega_{3}^{\prime}=1, \quad \omega_{2}^{\prime}, \quad \omega_{3}^{\prime} \geq 0
\end{aligned}
$$

where $y_{i^{g}}^{2}{ }^{\prime}$ and $y_{i^{9}}^{3}{ }^{\prime}$ separately denote the standardized value of delivery deadlines and products expiration dates that are calculated using Equations (17) $(19) ; \omega_{2}^{\prime}$ and $\omega_{3}^{\prime}$ respectively represent the weight coefficient of the delivery deadline and expiration dates; $n$ is the number of customers in the dynamic group $g_{i j}$. Based on the survey data that the company collects from its customers, there is a conclusion that customers attach more attention to the delivery deadline than to the expiration dates. So $\omega_{2}^{\prime}$ and $\omega_{3}^{\prime}$ are set to be 0.6 and 0.4 in this study.

$$
\begin{aligned}
y_{i^{g}}^{k} & =\frac{y_{i^{g}}^{k}-\bar{y}_{i^{g}}^{k}}{\mu_{i^{g}}^{k}} \\
\bar{y}_{i^{g}}^{k} & =\frac{\sum_{i=1}^{N} y_{i^{g}}^{k}}{N} \\
\mu_{i^{g}}^{k} & =\frac{\sum_{i=1}^{N}\left(y_{i^{g}}^{k}-\bar{y}_{i^{g}}^{k}\right)}{N-1}
\end{aligned}
$$

where $\bar{y}_{i^{g}}^{k}$ and $\mu_{i^{g}}^{k}$ are the values of the mean and standard deviation with respect to attribute $k$, and $N$ represents the total number of customers in one processing period.

\section{Case Analysis}

In this section, we use a real case to demonstrate the proposed method. The company is one of the largest groups within liquid milk producing in China, and the production capacity of dairy products reaches 6 million tons per year. The group has established over 20 production bases in 15 provinces of China. The products cover all Chinese market and are also exported to overseas market, but our study mainly focuses on the domestic distribution of the liquid milk. Relying on the supreme quality of its products, the company has many regular customers including $20 \%$ large customers as well as $80 \%$ small customers. When customer orders arrive, 
the planning and scheduling department need to determine the delivery dates and delivery producers. In accordance with the processed orders, the logistics department should arrange the transportation schedule. The manual scheduling of distribution system used by the company always results in highly inefficient schedules. Meanwhile, the demand characteristics of small customers are low quantities and high frequencies, which usually bring higher LTL (less than a truck load) transportation cost or delivery delay in order to achieve TL (a truck load) transportation, and the waste of transportation resources. Moreover, the delivery delay is always accompanied by the lower customer satisfaction.

\subsection{The Static Clustering and Dynamic Fuzzy Clustering}

In order to solve these problems, we choose one of the distribution areas as the study object, and Figure 2 shows the locations of 5 producers and 120 customers. Conventional clustering methods generally directly use the straight-line distance between two customers calculated according to their coordinates. However, there is always no feasible road along the shortest straight line in the actual situation. We adopt the real road distance to conduct the static clustering, and the data are collected from GIS or provided by ERP in the company. Clustering results are shown in Figure 2.

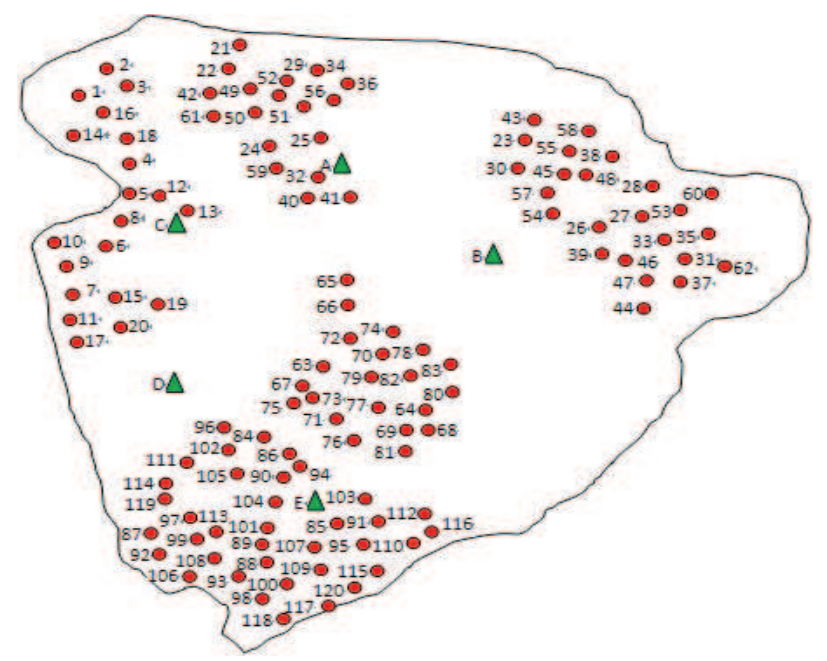

Figure 2 The proposed methodology framework

The company's order processing period is 3 days. We obtain the customers' order data from the order database of ERP in the company, which are scattered in April 1 to 6, 2009. According to the above procedure, all orders are processed in the above two continuous periods, including 51 customers and 49 customers respectively. In two different service periods, there may be the same customers or not, which all depend on the customers' order frequency.

According to the proposed dynamic clustering strategy, the calculation results are obtained by Matlab 7.8, as shown in Tables 2 and 3. Some customers appear in the two service periods, such as 24, 25, 32, 85, 95, 103, and 104. 
Table 2 The results of clustering in service period $T$

\begin{tabular}{ccccc}
\hline $\begin{array}{c}\text { Static } \\
\text { group }\end{array}$ & $\begin{array}{c}\text { Dynamic } \\
\text { group }\end{array}$ & $\begin{array}{c}\text { Customers } \\
\text { group }\end{array}$ & $\begin{array}{c}\text { Dynamic } \\
\text { ranking }\end{array}$ & $\begin{array}{c}\text { Producer service } \\
\text { priority }\end{array}$ \\
\hline \multirow{2}{*}{ G1 } & G11 & $1,2,3,4,14,16,18$ & 2 & C,AD,E,B \\
& G12 & $5,6,8,9,10,12,13$ & 5 & \\
G2 & G21 & $24,25,32,40,41,59$ & 1 & A,B,C,D,E \\
G3 & G31 & $23,30,38,45,48,55,57$ & 4 & B,A,C,D,E \\
& G32 & $26,27,33,39,46,47$ & 6 & \\
G5 & G51 & $84,86,90,94,96,102,105,111,114,119$ & 3 & E,D,C,B,A \\
& G52 & $85,91,95,103,104,110,112,116$ & 7 & \\
\hline
\end{tabular}

Table 3 The results of clustering in service period $T+1$

\begin{tabular}{|c|c|c|c|c|}
\hline $\begin{array}{l}\text { Static } \\
\text { group }\end{array}$ & $\begin{array}{c}\text { Dynamic } \\
\text { group }\end{array}$ & Customers & $\begin{array}{c}\text { Dynamic group } \\
\text { ranking }\end{array}$ & $\begin{array}{c}\text { Producer service } \\
\text { priority }\end{array}$ \\
\hline G1 & G11 & $7,11,15,17,19,20$ & 7 & $\mathrm{C}, \mathrm{A}, \mathrm{D}, \mathrm{E}, \mathrm{B}$ \\
\hline \multirow[t]{2}{*}{ G2 } & G21 & $21,22,29,34,36,42,49$ & 3 & $\mathrm{~A}, \mathrm{~B}, \mathrm{C}, \mathrm{D}, \mathrm{E}$ \\
\hline & $\mathrm{G} 22$ & $24,25,32,50,51,61$ & 2 & \\
\hline \multirow[t]{2}{*}{ G4 } & G41 & $63,67,71,73,75$ & 6 & E,D,B,C,A \\
\hline & G42 & $64,68,69,76,77,80,81$ & 5 & \\
\hline \multirow[t]{2}{*}{ G5 } & G51 & $87,92,97,99,106,108,104$ & 1 & $\mathrm{E}, \mathrm{D}, \mathrm{C}, \mathrm{B}, \mathrm{A}$ \\
\hline & G52 & $85,88,93,95,100,101,103,107,115,117,118,120$ & 4 & \\
\hline
\end{tabular}

Table 4 The results of routs construction in service period $T$

\begin{tabular}{cccccc}
\hline Producer & $\begin{array}{c}\text { Dynamic } \\
\text { group }\end{array}$ & $\begin{array}{c}\text { Optimal } \\
\text { route }\end{array}$ & $\begin{array}{c}\text { Vehicle } \\
\text { loading rate }\end{array}$ & $\begin{array}{c}\text { Travel } \\
\text { distance cost }\end{array}$ & $\begin{array}{c}\text { Total } \\
\text { cost }\end{array}$ \\
\hline A & G21 & $0-24-25-32-40-41-59-0$ & $98.21 \%$ & 531 & 531 \\
B & G31 & $0-57-38-48-30-23-55-45-0$ & $99.64 \%$ & 1085 & 2726 \\
& G32 & $0-47-39-26-27-33-46-0$ & $94.64 \%$ & 1641 & 4186 \\
C & G11 & $0-14-16-2-1-3-18-4-0$ & $96.07 \%$ & 1261 & 1689 \\
& G12 & $0-13-12-5-8-10-9-6-0$ & $99.64 \%$ & 2925 & 1179 \\
\\
E & G51 & $0-84-114-119-102-96-90-0$ & $98.57 \%$ & & \\
& \multirow{2}{*}{ G52 } & $0-94-111-105-86-0$ & $97.86 \%$ & 510 & \\
\hline
\end{tabular}




\subsection{Routs Construction}

We use the basic GA (genetic algorithms) to construct optimal route for each dynamic group. A fleet of vehicles with the same capacity $Q$ is set out for distribution service for customers from producers, and $Q$ is equal to 28t. The results are summarized in Tables 4 and 5. Note that in the service period $T+1$, based on the dynamic group ranking and the producer service priority, the dynamic group G42 is served by producer E due to the restriction of its production capacity. The vehicle loading rate can reach to $100 \%$ because gaps in the truck are not considered.

Table 5 The results of routs construction in service period $T+1$

\begin{tabular}{cccccc}
\hline Producer & $\begin{array}{c}\text { Dynamic } \\
\text { group }\end{array}$ & $\begin{array}{c}\text { Optimal } \\
\text { route }\end{array}$ & $\begin{array}{c}\text { Vehicle } \\
\text { loading rate }\end{array}$ & $\begin{array}{c}\text { Travel } \\
\text { distance cost }\end{array}$ & $\begin{array}{c}\text { Total } \\
\text { cost }\end{array}$ \\
\hline \multirow{2}{*}{ A } & G21 & $0-36-34-29-21-22-42-49-0$ & $98.21 \%$ & 1045 & 2013 \\
& G22 & $0-25-51-50-61-24-32-0$ & $97.50 \%$ & 968 & \\
C & G11 & $0-7-11-17-20-19-15-0$ & $99.29 \%$ & 1435 & 1435 \\
E & G51 & $0-104-97-87-92-106-108-99-0$ & $98.57 \%$ & 757 & 4468 \\
& G52 & $0-85-95-115-120-117-118-0$ & $98.57 \%$ & 1587 & \\
& & $0-101-88-93-100-107-103-0$ & $97.14 \%$ & & \\
& G42 & $0-76-77-80-64-68-69-81-0$ & $97.86 \%$ & 2124 & \\
& G41 & $0-71-73-75-67-63-0$ & $100 \%$ & 785 & 785 \\
\hline
\end{tabular}

\subsection{Comparison Between the Proposed Method and Direct Distribution Method}

In order to demonstrate the potential advantage of the proposed method, we compare the performance of the dynamic fuzzy clustering with the original strategy executed by the company. The results are presented in Table 5. The total cost of one producer is the sum of two continuous service periods. As can be seen in Table 5, the total cost of one producer is reduced by about $35 \%$, up to $38.09 \%$. The vehicle loading rates are also greatly improved, almost all above $95 \%$. So, the proposed method can provide the substantial benefits for the company.

\section{Conclusion}

This paper considers a large-scale distribution network encountered by a large milk producing company in China. The company is currently faced with raising LTL transportation cost, falling customer service levels and wasting delivery resources as a result of poor scheduling and routing decisions. We have proposed an efficient and effective dynamic fuzzy clustering method to solve the problem, including static clustering, order processing, and dynamic clustering, which is based on both the real delivery distance and order attributes. Significant improvement has been observed by using this method. Furthermore, the dynamic fuzzy clustering method cannot only be applied to this company who has multi-producers and multi-customers, but also to any large-scale and quick-responsive logistics distribution system. 


\section{References}

[1] Li Z P, Jiang S. Client grouping in distribution system optimization. Journal of Southwest Jiaotong University, 2006, 41(5): 594-598 (in Chinese).

[2] Zhu L, Li B. Improved clustering method based on artificial immune. Journal of Systems Engineering and Electronics, 2010, 21(6): 1111-1115.

[3] Yang Z, Chen H, Chu F. A Lagrangian relaxation approach for a large scale new variant of capacitated clustering problem. Computers \& Industrial Engineering, 2011, 61(2): 430-435.

[4] Nananukul N. Clustering model and algorithm for production inventory and distribution problem. Applied Mathematical Modelling, 2013, 37(24): 9846-9857.

[5] Lu Y R, Yang Y S, Lu F. Optimal vehicle routing problem based on fuzzy clustering analysis. Journal of Jilin University, 2006, 36(2): 147-151 (in Chinese).

[6] Hu T L, Sheu J B. A fuzzy-based customer classification method for demand-responsive logistical distribution operations. Fuzzy Sets and Systems, 2003, 139(2): 431-450.

[7] Sheu J B. A novel dynamic resource allocation model for demand-responsive city logistics distribution operations. Transportation Research Part E, 2006(42): 445-472.

[8] Kuo R J, Hu T L, Sheu J B, et al. Application of fuzzy clustering based approach for customer classification in logistical operations. 2006 IIE Annual Conference and Exposition, Institute of Industrial Engineers, 2006, $20-24$.

[9] Sheu J B. A hybrid fuzzy-optimization approach to customer grouping-based logistics distribution operations. Applied Mathematical Modeling, 2007, 31(6): 1048-1066.

[10] Liu C S, Lai M Y. The fuzzy clustering and vehicle coordination strategy to stochastic vehicle routing problem. Journal of Industrial Engineering/Engineering Management, 2010, 24(2): 75-78.

[11] Qi M Y, Ding G X, Zhou Y, et al. Vehicle routing problem with time windows based on spatiotemporal distance. Journal of Transportation Systems Engineering and Information Technology, 2011, 11(1): 85-89.

[12] Yvcenur G N, Demirel N C. A new geometric shape-based genetic clustering algorithm for the multi-depot vehicle routing problem. Expert Systems with Applications, 2011, 38(9): 11859-11865.

[13] Wang Y, Ma X, Lao Y, Wang Y. A fuzzy-based customer clustering approach with hierarchical structure for logistics network optimization. Expert Systems with Applications, 2014, 41(2): 521-534.

[14] Yin X F, Li P K, Chong Y T. A fuzzy c-means based hybrid evolutionary approach to the clustering of supply chain. Computers \& Industrial Engineering, 2013, 66(4): 768-780.

[15] Li Z, Wong W K, Kwong C K. An integrated model of material supplier selection and order allocation using fuzzy extended AHP and multiobjective programming. Mathematical Problems in Engineering, 2013, $1-14$.

[16] Chen S J, Chen S M. Fuzzy risk analysis based on similarity method for demand-responsive logistical distribution operations. Fuzzy Sets and System, 2003, 139(3): 431-450. 